\title{
PROYECTO M.A.T.R.I.X.: MODALIDADES DE APRENDIZAJE TELEMÁTICO Y RESULTADOS INTERUNIVERSITARIOS EXTRAPOLABLES AL BLENDED LEARNING
}

\author{
(PROJECT M.A.T.R.I.X.: MODALITIES OF TELEMATIC LEARNING AND INTERUNIVERSITY \\ RESULTS EXTRAPOLABLES TO THE BLENDED LEARNING)
}

\author{
$\mathrm{M}^{\mathrm{a}}$ Esther del Moral Pérez \\ Lourdes Villalustre Martínez \\ Universidad de Oviedo (España)
}

\section{RESUMEN}

Con el Proyecto MATRIX (Modalidades de Aprendizaje Telemático y Resultados Interuniversitarios eXtrapolables al blended learning $)^{1}$ se han identificado y descrito la diversidad de prácticas metodológicas docentes adoptadas en una muestra representativa de asignaturas virtuales y semipresenciales de diferentes titulaciones impartidas en distintas universidades españolas integradas en el Campus Virtual Compartido del G9, con la intención de extrapolar aquellas experiencias consideradas como "buenas prácticas", tanto por la calidad de sus diseños pedagógicos, tras haber sido sometidas a una evaluación por parte de expertos; como por el nivel de satisfacción suscitados en los estudiantes que las han cursado; como por su contribución efectiva al desarrollo de las competencias específicas y genéricas de cada titulación; etc..., a los nuevos contextos de aprendizaje, propuestos desde el EEES, caracterizados por su apuesta por una metodología y/o aprendizaje mixto (blended learning).

Palabras clave: blended learning, indicadores de calidad, buenas prácticas, satisfacción de los estudiantes.

\section{ABSTRACT}

The project M.A.T.R.I.X. has enabled identification of the diversity of educational methods adopted in virtual and mixed (blended) subjects used by Spanish universities integrated in the Virtual Shared Campus of the G9. The purpose of this study is to extrapolate from those experiences considered "good practices" in new and mixed learning modalities, as proposed by the European Space for Higher 
Education, in terms of quality of pedagogical design, level of student satisfaction, and contribution to the development of generic and specific competiencies.

Keywords: blended learning, indicators of quality, good practices, satisfaction of the students.

\section{EL PROYECTO M.A.T.R.I.X.}

El proyecto M.A.T.R.I.X. (Modalidades de Aprendizaje Telemático y Resultados Interuniversitarios eXtrapolables al blended learning) fue financiado por el Ministerio de Educación y Ciencia a través del Programa de Estudios y Análisis, se desarrolló durante el curso académico 2007/2008, implicando a una veintena de investigadores. En él se llevó a cabo un proceso de evaluación de una muestra de catorce asignaturas de diferentes titulaciones impartidas total o parcialmente en entornos virtuales, pertenecientes a cinco universidades españolas como son la Universidad de Oviedo, la Pública de Navarra, la del País Vasco, la de Extremadura y la de Zaragoza, todas ellas integradas en el Campus Virtual Compartido (CVC) del G9.

\section{Objetivo del proyecto MATRIX}

Determinar los indicadores de calidad técnica y didáctica que deben tenerse en cuenta en el diseño de asignaturas virtuales o presenciales impartidas con apoyos virtuales, a partir de:

- Las creación de un mapa con la diversidad de prácticas formativas llevadas a cabo en las asignaturas integradas en MATRIX, a tenor de los informes descriptivos que los docentes, responsables de las mismas, elaboraron visibilizando los criterios metodológicos y técnicos que las rigen.

- El análisis cualitativo, de la muestra de catorce asignaturas (virtuales y semipresenciales), llevado a cabo por expertos en diseño e implementación de proyectos de e-learning, con la intención de extrapolar aquellas experiencias consideradas como "buenas prácticas", -tanto por la calidad de sus diseños pedagógicos y técnicos como por su contribución efectiva al desarrollo de las competencias específicas y genéricas de cada titulación-, a los nuevos contextos de aprendizaje, propuestos desde el EEES, caracterizados por su apuesta por 
una metodología semipresencial y/o aprendizaje mezclado (blended learning) apoyado en el uso de entornos virtuales.

- Las valoraciones de una muestra de 290 universitarios que han cursado asignaturas virtuales dentro del CVC del G9, y su nivel de satisfacción sobre los entornos tecnológicos en los que aprenden, obtenidos a través de un cuestionario de opinión. Infiriéndose las propuestas de mejora, que desde su condición de usuarios, demandan.

\section{Fases del Proyecto MATRIX}

En un primer momento, se elaboró un mapa con la diversidad de prácticas metodológicas docentes que se adoptaron en la muestra de asignaturas, -consideradas como referentes de "buenas prácticas"-, ofertadas por las diferentes universidades españolas que formaron parte del proyecto, a partir de las descripciones que realizaron los propios docentes responsables de las mimas, haciendo visible tanto el diseño pedagógico que las orienta, como el modo en que contribuyen efectivamente al desarrollo de las competencias genéricas y específicas de cada titulación.

Se definieron los indicadores que sirven para identificar las prácticas formativas de calidad soportadas en entornos virtuales, los cuales inciden sobre las pautas pedagógicas y técnicas que contribuyen a facilitar tanto el trabajo autónomo de los estudiantes como el grupal, reforzando el desarrollo de competencias de diversa índole. Tras la enumeración de éstos, se construyó un instrumento de evaluación en el que se recogían los principales elementos de valoración referidos a la presentación del entorno y de los materiales, a la pertinencia del diseño instructivo adoptado y a la acción tutorial dispensada por los docentes en ellas. Se estableció que cada materia debía ser analizada al menos por tres evaluadores externos, y dicho instrumento de evaluación fue el que se utilizó para llevar a cabo los análisis DAFO (Debilidades, Amenazas, Fortalezas y Oportunidades) de las catorce asignaturas que integraban el proyecto, con objeto de identificar las fortalezas y debilidades de las mismas, y poder enunciar las propuestas de mejora correspondientes en cada caso.

En último término, se recogieron las valoraciones de una muestra de 290 estudiantes, -matriculados en alguna de las asignaturas impartidas en el CVC del G9-, sobre las metodologías que adoptan las asignaturas que cursaron, medidas en relación tanto a su consideración sobre la organización y estructura de los contenidos, la acción tutorial que en ellas se llevó a cabo, como sobre las actividades grupales e 
individuales que se desarrollaron y las fórmulas de evaluación que se adoptaron en las mismas.

De este modo, y a la vista de lo expuesto, en el proyecto MATRIX se desarrollaron diferentes actuaciones que giraban alrededor a tres ejes fundamentales:



Grafico 1. Ámbitos de actuación desarrollados en el proyecto M.A.T.R.I.X.

Con el fin de facilitar la consecución de los objetivos propuestos en el proyecto M.A.T.R.I.X. se creó un espacio de trabajo sustentado bajo una plataforma de $e$ learning, en la cual se diseñó un entorno multimedia e interactivo que sirvió de soporte para la realización de las diferentes tareas de carácter colaborativo que debían desarrollar los docentes-investigadores participantes, a partir de las cuales se recabaron aportaciones de gran interés, y se extrajeron conclusiones significativas.

La creación de esta comunidad virtual permitió el intercambio de experiencias e innovaciones docentes entre participantes en la investigación, quienes han tenido la oportunidad de ofrecer sus impresiones, expectativas, demandas y soluciones sobre aspectos concretos, tales como: el diseño de materiales didácticos, la organización y presentación de los mismos, la planificación de actividades formativas, la evaluación, etc..., a través de los foros y talleres que se habilitaron a lo largo de la ejecución del proyecto. 


\section{Descripción del entorno MATRIX}

El entorno virtual proporcionó diversos espacios de trabajo a través de una interfaz gráfica sencilla e intuitiva basada en la metáfora cinematográfica de la conocida película MATRIX. En él se albergaron diferentes recursos orientados a facilitar a los participantes tanto las tareas individuales como el trabajo colaborativo planteado en el proyecto. Para lo cual, se incorporó en el entorno virtual una guía de trabajo, en donde se explicitaban los objetivos de éste, la metodología a seguir, las actividades que debían realizar, etc.; también se habilitó un calendario para fijar las fechas claves, a modo de agenda de trabajo; un glosario de términos con el fin de clarificar los que estaban relacionados con la temática del proyecto, y que por su complejidad conceptual pudieran inducir a diferentes interpretaciones; así como foros de debate y buzón de correo electrónico para facilitar la comunicación entre los investigadores y el desarrollo de las actividades colaborativas planteadas.

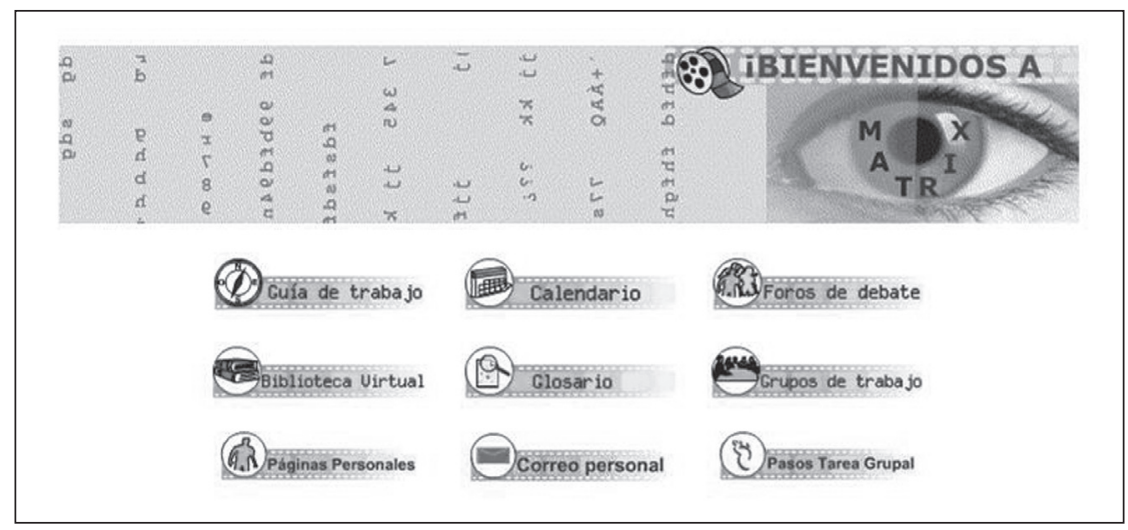

Figura 1. Interfaz de entrada al entorno virtual del proyecto

Igualmente, se diseñaron materiales multimedia orientados a ofrecer pautas y recursos que guiasen el trabajo colaborativo y la reflexión compartida entre los docentes implicados en el proyecto. Para ello, se creó una biblioteca virtual que recopilaba documentación de diversa índole; y una Webquest, a través de la cual se presentaba, de manera interactiva, los pasos a seguir para el desarrollo de las actividades grupales.

La biblioteca virtual proporcionaba referencias bibliográficas y acceso a materiales "on line" relacionados con la temática del proyecto, a través de un listado 
de libros y revistas agrupados en función de cuatro áreas: Convergencia Europea, Competencias, Evaluación y Metodología docente. En ella, se recopilan archivos procedentes de organismos e instituciones oficiales, tales como la ANECA, el Ministerio de Educación, etc.; así como artículos, actas de congresos y jornadas, al igual que revistas electrónicas como Pixel-Bit, Instructional Technology of Distance Learning, etc.

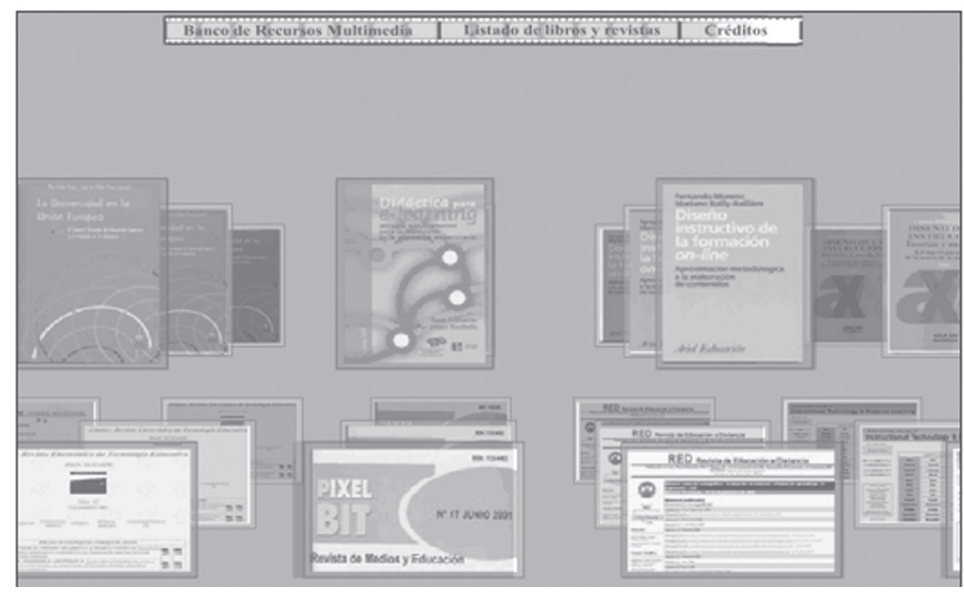

Figura 2. Biblioteca Virtual realizada para el proyecto M.A.T.R.I.X.

Así pues, se adoptó una metodología de trabajo colaborativo basada en el uso de Webquest (Dodge, 1995) a través de la cual se especificaban la estructura y los pasos que tenían que seguir los participantes en el proyecto. Para ello, se creó una presentación en la que a través de tres enlaces se detallaba el tipo de tarea que debían realizar individual o colaborativamente, en concreto, se trataba de un análisis descriptivo de las prácticas docentes, materiales didácticos, ..., que cada investigador empleaba en su asignatura; también se les ofrecían claramente los pasos a seguir o pautas procedimentales para llevar a cabo dichos análisis descriptivos, y de este modo, garantizar un mínimo de homogeneidad en las descripciones solicitadas a todos los docentes, facilitándoles algún ejemplo de ficha para su elaboración, y los recursos disponibles para ello. 


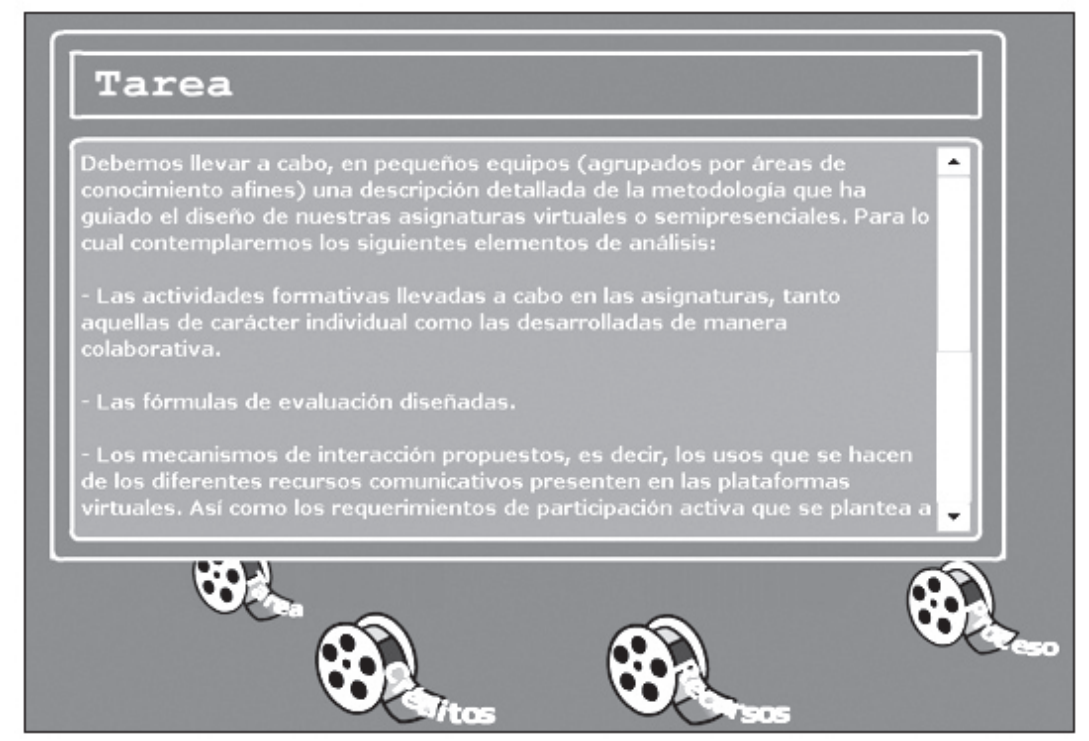

Figura 3. Presentación del trabajo grupal a través de Webquest

Mediante la utilización de los recursos multimedia y con la participación activa de los miembros del proyecto en las dinámicas propuestas en el entorno virtual creado al efecto, -sustentado en una plataforma de e-learning-, se desarrolló el proyecto M.A.T.R.I.X., en el cual convergían diferentes metodologías de investigación a partir de las cuales se obtuvieron datos tanto de carácter cualitativo como cuantitativo de gran interés, que sirvieron para vislumbrar el objetivo principal del estudio.

\section{Metodologías de investigación que convergen en MATRIX}

Primeramente, se adoptó una metodología descriptiva próxima al estudio etnográfico (Goetz y Lecompte, 1988), puesto que los docentes individual o colectivamente debían elaboran un informe descriptivo sobre el diseño instructivo que orientaba su actividad docente apoyada total o parcialmente en el uso de entornos virtuales. Dicho informe debía ajustarse a unas pautas comunes que contemplan entre otros aspectos, los objetivos que persigue la asignatura de la que son responsables, los contenidos que en ella se desarrollan, las actividades que se plantean, la evaluación llevada a cabo, la acción tutorial dispensada, etc..., los cuales, además debían estar suficientemente ilustrados con "pantallazos" tanto del entorno, como de la presentación de los contenidos y recursos más representativos de la misma. 
Así, a través de un ejercicio de introspección y análisis de su propia práctica, cada docente daba a conocer al resto la metodología de su asignatura y facilitaba su comprensión, dado que dichos informes se ponían a disposición de todos los miembros de la Comunidad MATRIX para que sirvieran de referente, y guiaran, más tarde, la observación de los evaluadores externos, quienes procederían a identificar los puntos fuertes y débiles (análisis DAFO) de cada asignatura.

En este sentido, esta metodología descriptiva, próxima al estudio etnográfico, proporcionó una serie de datos cualitativos de gran valor, al exigir que cada investigador se sumergiera en sus propias asignaturas virtuales o semipresenciales elaborando un exhaustivo informe previamente pautado, que permitiera visibilizar los principios metodológicos que guiaban el desarrollo formativo de cada materia. Dichos informes constituyeron una interesante fuente de información que sirvió para elaborar el mapa con la diversidad de prácticas formativas tanto de carácter individual como grupal desarrolladas en la muestra de asignaturas que nos ocupa.

Además de los datos obtenidos a partir de las aportaciones personales realizadas por cada docente, mediante los mencionados informes descriptivos de sus propias asignaturas, se estableció que cada materia debía se analizada de forma externa, al menos por tres evaluadores -integrantes del proyecto MATRIX-. Y se estimó pertinente optar por la metodología del análisis DAFO para la identificación de debilidades, amenazas, fortalezas y oportunidades de cada una de las catorce asignaturas que formaban parte del proyecto. Ello implicó la creación de un instrumento de observación y evaluación común que recogiera los tres bloques objeto de análisis, tales como el diseño técnico de los materiales, el diseño instructivo y la acción tutorial dispensada.

Así pues, el pormenorizado estudio de casos, -según Bogdan y Biklen, 1982-, materializado en el examen sistemático, detallado, intensivo, profundo e interactivo de cada asignatura realizado de forma individual por los evaluadores, orientado a señalar tanto sus fortalezas como debilidades, permitió finalmente y de forma conjunta, enunciar las propuestas de mejora pertinentes para garantizar el éxito en los procesos formativos apoyados en entornos virtuales.

Por otro lado, se obtuvieron interesantes datos cuantitativos a partir de un cuestionario destinado a valorar el nivel de satisfacción de los estudiantes universitarios que cursaron asignaturas en su modalidad virtual dentro del CVC del G9, medido a través de su valoración sobre los contenidos didácticos, las actividades propuestas, el sistema de evaluación, la acción tutorial, las habilidades comunicativas del docente, ..., entre otros aspectos. 
En un intento de presentar de forma organizada y sistemática los resultados derivados de las diferentes fases llevadas a cabo a lo largo del proyecto MATRIX, procedemos a sintetizarlos de forma parcelada.

\section{PRESENTACIÓN DE RESULTADOS}

\section{Datos cualitativos}

Mapa con la diversidad de prácticas docentes desarrolladas en MATRIX

Como ya se ha mencionado, en el proyecto se analizaron un total de 14 asignaturas de diferentes titulaciones de cinco universidades españolas que forman parte del CVC del G9. Cada docente elaboró un breve informe explicando los principios metodológicos que regían su asignatura virtual o semipresencial en relación a los objetivos que se persiguen con la misma; las competencias genéricas (instrumentales, interpersonales y sistémicas) que con ella se pretenden consolidar; los contenidos que se desarrollan; las actividades que se plantean, -tanto individuales como grupales-; la evaluación llevada a cabo; la acción tutorial promovida; etc... Fruto de dichos informes se logró establecer un mapa identificando la diversidad de prácticas formativas que la muestra de asignaturas integradas en MATRIX adoptan.

Asimismo, y tras el análisis DAFO de cada una de ellas, se hicieron visibles las consideradas como referentes de "buenas prácticas docentes" con la intención de dar a conocer aquellas que pueden suponer un modelo a seguir. En este sentido, y desde una perspectiva pedagógica entendemos, -al igual que Epper y Bates (2004)-, que una materia puede ser considerada como referente de "buena práctica" cuando contribuye a mejorar el desarrollo de un proceso formativo desde un enfoque innovador basado en la búsqueda de la excelencia, y por ello puede considerarse extrapolable a otros contextos.

En M.A.T.R.I.X. se han descrito diversas asignaturas consideradas como "buenas prácticas" cuyas innovaciones docentes apoyadas en los entornos virtuales contribuyen a la mejora cualitativa del proceso de enseñanza-aprendizaje. A continuación se presenta gráficamente (Gráficos 2 y 3) este mapa que recoge la formulación de un total de ocho prácticas de carácter individual, y otras ocho de carácter grupal, identificando en cada caso el tipo de competencias que se pueden llegar a desarrollar y/o consolidar con cada una de ellas. 


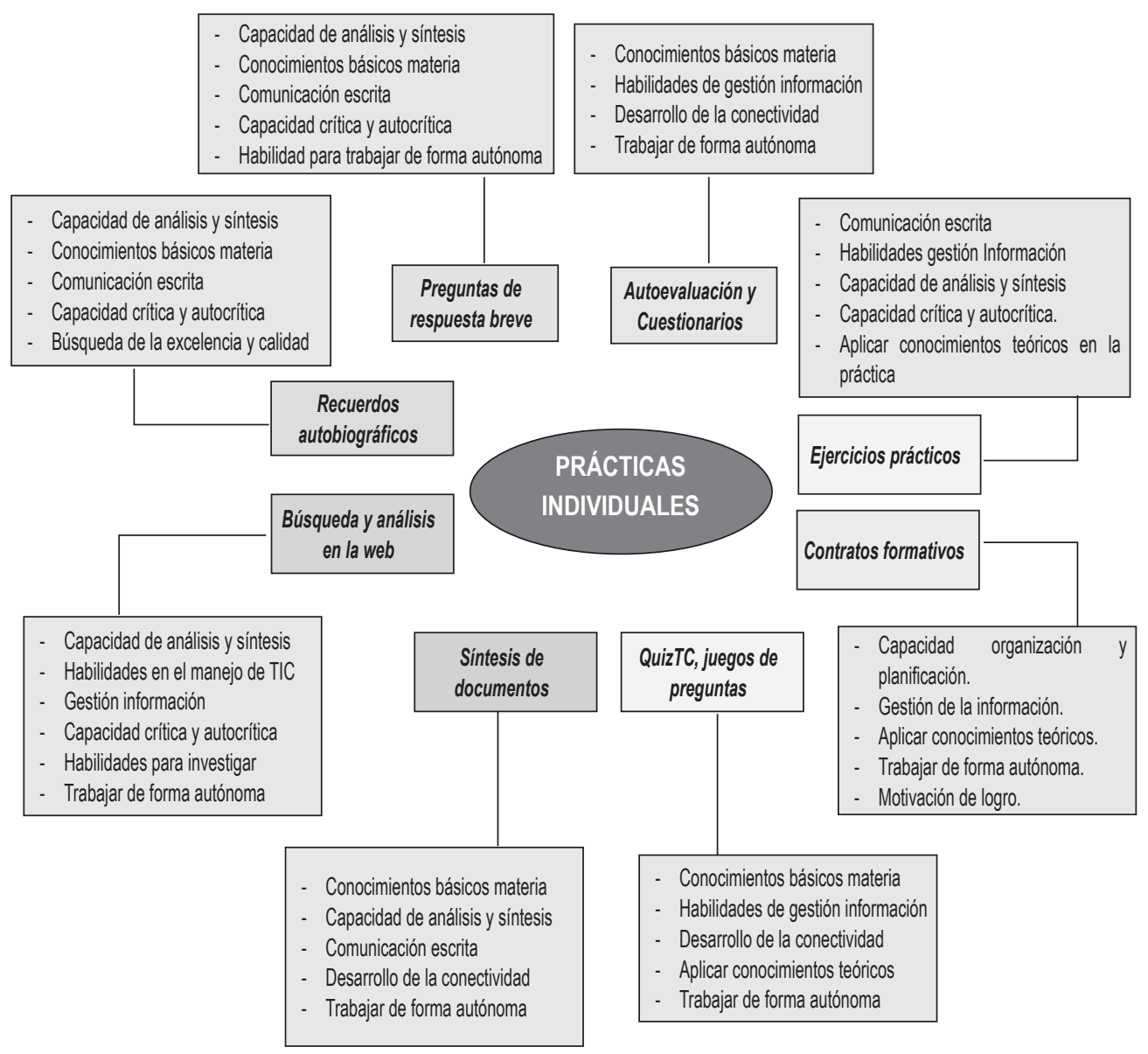

Grafico 2. Mapa con la diversidad de prácticas individuales y las competencias transversales genéricas que en ellas se desarrollan 


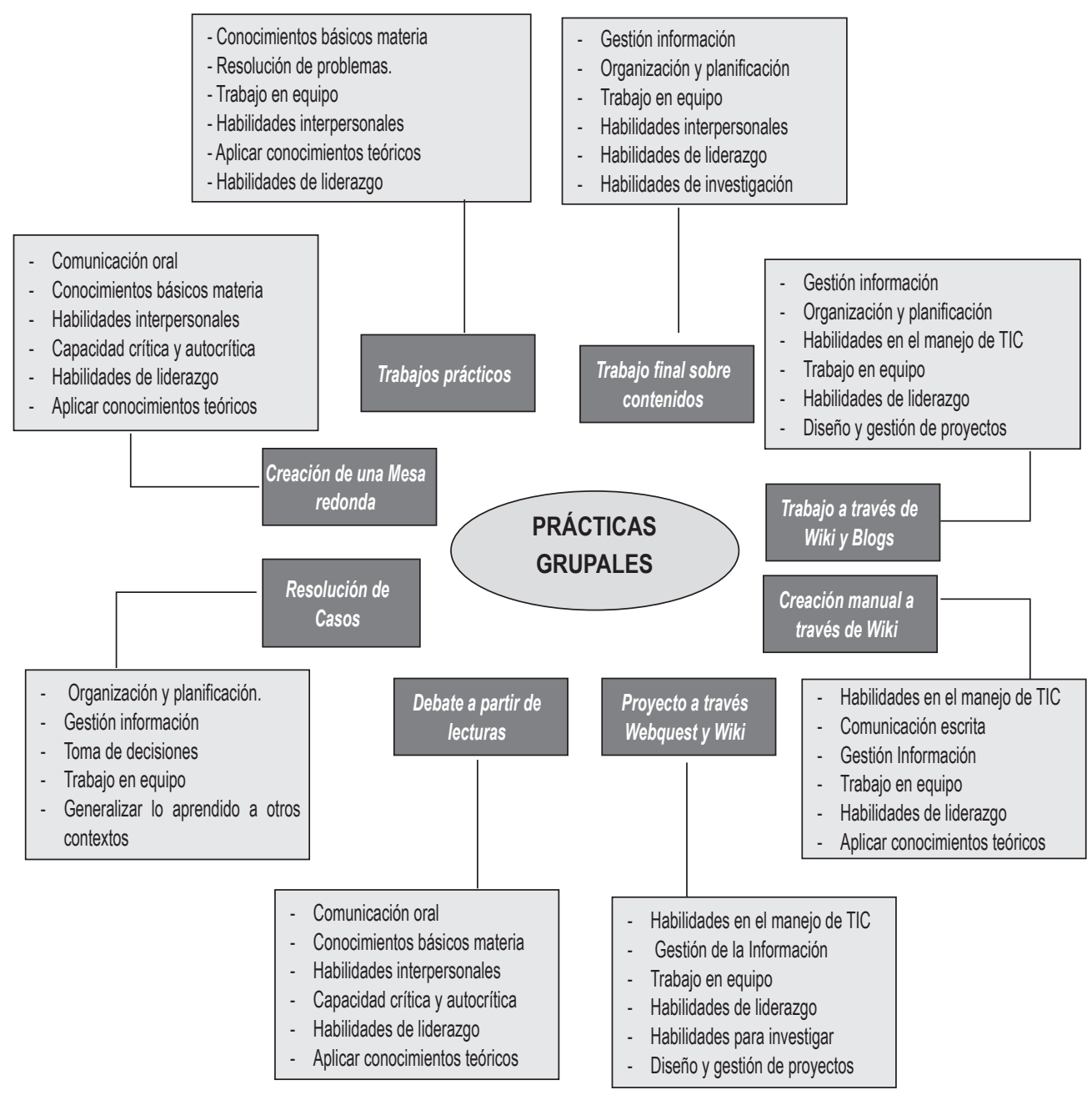

Grafico 3. Mapa con la diversidad de prácticas grupales y las competencias transversales genéricas que en ellas se desarrollan

Las prácticas propuestas, tanto grupales como individuales, promueven el desarrollo y adquisición de competencias de carácter genérico, como queda reflejado en los gráficos 2 y 3. No obstante, debemos señalar que la planificación de las diferentes actividades que tienen como fin la formación integral del estudiante mediante la potenciación y adquisición de diferentes competencias, a través de una metodología mixta o "blended learning", supone un nuevo reto para la práctica 
pedagógica de los docentes, puesto que han de reformular el modelo de enseñanza que hasta el momento venían aplicando. En este sentido, las prácticas que desde el proyecto MATRIX se analizaron han experimentado una evolución progresiva hacia metodologías más participativas, centradas en el discente y apoyadas en el uso de herramientas tecnológicas, constituyendo un amplio repertorio de "buenas prácticas" docentes.

Mediante la incorporación de actividades colaborativas e individuales se potencian y desarrollan toda una serie de competencias de carácter instrumental, interpersonal y sistémico en los discentes, atendiendo a los nuevos requerimientos impulsados por el proceso de Convergencia Europea. Dicho proceso implica ciertos cambios que afectan tanto a la estructura organizativa de los planes de estudio, como a la metodología docente, a las prácticas evaluativas, al sistema de tutorización de los estudiantes, etc., lo que ha provocado que muchos docentes modifiquen sus asignaturas, adoptando una nueva metodología centrada en el discente, que conlleva una reasignación de los roles a desempeñar por los distintos agentes implicados en el proceso formativo.

En este sentido, los estudiantes deben desarrollar nuevas competencias que supongan una actitud más activa y comprometida con su propio aprendizaje, en permanente adaptación a los cambios sociales, alentando su crecimiento intelectual y expansión de habilidades. Ese viraje en la forma de entender los procesos de enseñanza-aprendizaje da paso a un diseño pedagógico centrado en la figura del estudiante, facilitándole el trabajo autónomo y el colaborativo, así como la adquisición tanto de competencias específicas propias de su perfil profesional, como las competencias transversales o genéricas, categorizadas en instrumentales, interpersonales y sistémicas (Del Moral y Villalustre, 2008).

Hay que destacar que con la utilización de las plataformas de e-learning, los recursos tecnológicos que ofrece la red Internet y las cada vez más sofisticadas herramientas de la Web 2.o (social software), etc... (Del Moral, Cernea y Villalustre, 2007) se posibilita que los estudiantes adquieran todas aquellas competencias necesarias, no sólo para el ejercicio de una profesión concreta, sino también otras relativas al uso eficiente de las TIC, muy demandadas en la sociedad actual.

Análisis DAFO efectuado sobre las asignaturas de MATRIX

A continuación se presentan las valoraciones que los evaluadores realizaron sobre cada una de las catorce asignaturas de la muestra que nos ocupa, las cuales se 


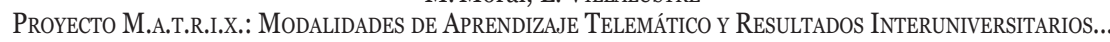

agruparon en función del área de conocimiento a la que pertenecían, de modo que las observaciones se formulan de forma genérica, identificando por un lado, en la Tabla 1 las fortalezas de las mimas, y en la Tabla 2 las debilidades.

\begin{tabular}{|c|c|c|c|}
\hline \multicolumn{4}{|c|}{ FORTALEZAS DETECTADAS EN LAS ASIGNATURAS MATRIX POR ÁREAS DE CONOCIMIENTO } \\
\hline \multirow{2}{*}{$\begin{array}{c}\text { Área de } \\
\text { Conocimiento }\end{array}$} & \multicolumn{3}{|c|}{ Aspectos objeto de análisis } \\
\hline & $\begin{array}{c}\text { Diseño Técnico de } \\
\text { los Materiales }\end{array}$ & Diseño Instructivo & Acción Tutorial \\
\hline Ciencias & $\begin{array}{l}\text { - Presentación clara } \\
\text { y ordenada de la } \\
\text { página principal de la } \\
\text { asignatura. } \\
\text { - Buena presentación } \\
\text { de los materiales } \\
\text { didácticos. } \\
\text { - Uso apropiado de } \\
\text { diferentes recursos } \\
\text { multimedia. }\end{array}$ & $\begin{array}{l}\text { - La guía didáctica describe } \\
\text { con precisión todos los } \\
\text { elementos curriculares. } \\
\text { - Se propicia la participación } \\
\text { de los discentes a través de } \\
\text { las actividades planificadas. } \\
\text { - Adecuada temporalización } \\
\text { de las actividades. }\end{array}$ & $\begin{array}{l}\text { - Comunicación fluida. } \\
\text { Prácticas grupales } \\
\text { a través de foros } \\
\text { temáticos. } \\
\text { - Asesoramiento } \\
\text { personalizado mediante } \\
\text { el correo interno de la } \\
\text { asignatura. }\end{array}$ \\
\hline $\begin{array}{l}\text { Ingeniería } \\
\text { y Tecnología }\end{array}$ & $\begin{array}{l}\text { - Presentación } \\
\text { interactiva de } \\
\text { los contenidos } \\
\text { formativos. } \\
\text { - Utilización de } \\
\text { diferentes recursos } \\
\text { multimedia. } \\
\text { - Incorporación de } \\
\text { un sistema de } \\
\text { navegación intuitivo. } \\
\text { - Contenidos } \\
\text { didácticos } \\
\text { presentados en } \\
\text { varios formatos. }\end{array}$ & $\begin{array}{l}\text { - Presentación de una guía } \\
\text { docente clara y detallada. } \\
\text { - Incorporación de materiales } \\
\text { complementarios. } \\
\text { - Utilización de contratos de } \\
\text { aprendizaje para fomentar la } \\
\text { motivación. } \\
\text { - Realización de una } \\
\text { evaluación contínua. } \\
\text { - Uso de herramientas } \\
\text { que facilitan el estudio } \\
\text { (Calendario, tablón de } \\
\text { avisos, etc.). } \\
\text { - Coherencia entre los } \\
\text { objetivos, contenidos, } \\
\text { actividades y la evaluación. }\end{array}$ & $\begin{array}{l}\text { - Fomento de la } \\
\text { participación. } \\
\text { - Creación de una activa } \\
\text { comunidad virtual de } \\
\text { aprendizaje. } \\
\text { - Empleo de un tono } \\
\text { cordial y asertivo en } \\
\text { las intervenciones } \\
\text { docentes. } \\
\text { - Desarrollo de prácticas } \\
\text { tutoriales tanto } \\
\text { individuales como } \\
\text { grupales. }\end{array}$ \\
\hline
\end{tabular}




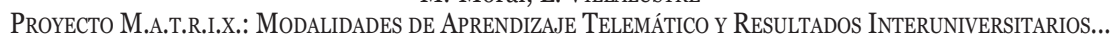

\begin{tabular}{|c|c|c|c|}
\hline \multicolumn{4}{|c|}{ FORTALEZAS DETECTADAS EN LAS ASIGNATURAS MATRIX POR ÁREAS DE CONOCIMIENTO } \\
\hline \multirow{2}{*}{$\begin{array}{c}\text { Área de } \\
\text { Conocimiento }\end{array}$} & \multicolumn{3}{|c|}{ Aspectos objeto de análisis } \\
\hline & $\begin{array}{c}\text { Diseño Técnico de } \\
\text { los Materiales }\end{array}$ & Diseño Instructivo & Acción Tutorial \\
\hline $\begin{array}{l}\text { Ciencias } \\
\text { Sociales y } \\
\text { Jurídicas }\end{array}$ & $\begin{array}{l}\text { - Empleo de una } \\
\text { presentación } \\
\text { multimedia a modo de } \\
\text { guía de estudio. } \\
\text { - Utilización de mapas } \\
\text { conceptuales para } \\
\text { la presentación } \\
\text { esquemática de los } \\
\text { contenidos. } \\
\text { - Utilización de } \\
\text { diferentes recursos } \\
\text { multimedia. } \\
\text { - Presentación clara } \\
\text { y ordenada de la } \\
\text { página principal de la } \\
\text { asignatura. } \\
\text { - Presentación } \\
\text { interactiva de los } \\
\text { contenidos formativos. }\end{array}$ & $\begin{array}{l}\text { - Realización de una evaluación } \\
\text { contínua a través de } \\
\text { entrevistas de seguimiento. } \\
\text { - Coherencia entre los } \\
\text { objetivos, contenidos, } \\
\text { actividades y la evaluación. } \\
\text { - Uso de herramientas } \\
\text { que facilitan el estudio } \\
\text { (Calendario, tablón de avisos, } \\
\text { etc.). } \\
\text { - Incorporación de ejercicios de } \\
\text { autoevaluación. } \\
\text { - Presentación de una guía } \\
\text { docente clara y detallada. } \\
\text { - Inclusión de materiales } \\
\text { complementarios. }\end{array}$ & $\begin{array}{l}\text { - Desarrollo de una tutoría } \\
\text { individual y grupal. } \\
\text { - Creación de una } \\
\text { comunidad virtual de } \\
\text { aprendizaje. } \\
\text { - Fomento de la } \\
\text { participación, sometida a } \\
\text { evaluación. } \\
\text { - Intervenciones docentes } \\
\text { cordiales. } \\
\text { - Creación de diferentes } \\
\text { foros temáticos. } \\
\text { - Fomento de la } \\
\text { participación, delegando } \\
\text { la coordinación de } \\
\text { los foros/wikis en los } \\
\text { discentes. }\end{array}$ \\
\hline $\begin{array}{c}\text { Ciencias } \\
\text { Biológicas y de } \\
\text { la Salud }\end{array}$ & $\begin{array}{l}\text { - Materiales didácticos } \\
\text { muy completos y de } \\
\text { buena calidad. } \\
\text { - Presentación clara } \\
\text { y ordenada de la } \\
\text { página principal de la } \\
\text { asignatura. }\end{array}$ & $\begin{array}{l}\text { - Incorporación de una guía } \\
\text { docente detallada. } \\
\text { - Coherencia entre los } \\
\text { elementos didácticos. } \\
\text { - Inclusión de materiales } \\
\text { complementarios. } \\
\text { - Utilización de ejercicios de } \\
\text { autoevaluación. }\end{array}$ & $\begin{array}{l}\text { - Fomento de la } \\
\text { participación a través } \\
\text { de las diferentes } \\
\text { herramientas de } \\
\text { comunicación presentes } \\
\text { en las asignaturas. }\end{array}$ \\
\hline
\end{tabular}

Tabla 1. Fortalezas de las asignaturas MATRIX, detectadas a partir de los análisis DAFO, considerando los diferentes aspectos objeto de estudio 
Proyecto M.A.T.R.I.X.: ModalidAdes De AprendiZAJE TelEMÁtico y Resultados InTERUNIVERSITARIOS...

\begin{tabular}{|c|c|c|c|}
\hline \multicolumn{4}{|c|}{ DEBILIDADES DETECTADAS EN LAS ASIGNATURAS MATRIX POR ÁREAS DE CONOCIMIENTO } \\
\hline \multirow{2}{*}{$\begin{array}{c}\text { Área de } \\
\text { Conocimiento }\end{array}$} & \multicolumn{3}{|c|}{ Aspectos objeto de análisis } \\
\hline & $\begin{array}{c}\text { Diseño Técnico de los } \\
\text { Materiales }\end{array}$ & Diseño Instructivo & Acción Tutorial \\
\hline Ciencias & $\begin{array}{l}\text { - Carencia de algún tipo de } \\
\text { mensajes para guiar al } \\
\text { estudiante en la página } \\
\text { principal de la asignatura. }\end{array}$ & $\begin{array}{l}\text { - Presentación de una } \\
\text { guía de estudio poco } \\
\text { precisa. }\end{array}$ & $\begin{array}{l}\text { - No se fomenta el trabajo } \\
\text { grupal, por lo que se sugiere } \\
\text { crear grupos de trabajo para } \\
\text { el desarrollo de actividades } \\
\text { grupales. }\end{array}$ \\
\hline $\begin{array}{l}\text { Ingeniería y } \\
\text { Tecnología }\end{array}$ & $\begin{array}{l}\text { - Presentación excesiva } \\
\text { de recursos en la página } \\
\text { principal de la asignatura. } \\
\text { - Falta de homogeneidad } \\
\text { de estilos en los iconos, } \\
\text { gráficos, etc., que llevan a la } \\
\text { confusión. } \\
\text { - Duplicidad de contenidos en } \\
\text { los materiales didácticos. } \\
\text { - Presentación de los } \\
\text { contenidos únicamente } \\
\text { en formato pdf para su } \\
\text { impresión. } \\
\text { - Exposición caótica de los } \\
\text { iconos que dan acceso a los } \\
\text { recursos y contenidos }\end{array}$ & $\begin{array}{l}\text { - Incorporación de } \\
\text { una guía de estudio } \\
\text { demasiado extensa y } \\
\text { poco precisa. } \\
\text { - Ausencia de fórmulas } \\
\text { que garanticen una } \\
\text { evaluación continua. } \\
\text { - Concepción, exclusiva, } \\
\text { de la asignatura virtual } \\
\text { como repositorio de } \\
\text { materiales y recursos. }\end{array}$ & $\begin{array}{l}\text { - Falta de coordinación por } \\
\text { parte del docente en los foros } \\
\text { telemáticos. } \\
\text { - Ausencia de una explotación } \\
\text { de los foros con valor } \\
\text { formativo. } \\
\text { - Improvisación en la } \\
\text { participación en los foros. }\end{array}$ \\
\hline $\begin{array}{l}\text { Ciencias } \\
\text { Sociales y } \\
\text { Jurídicas }\end{array}$ & $\begin{array}{l}\text { - Presentación de los } \\
\text { contenidos únicamente } \\
\text { en formato pdf para su } \\
\text { impresión. } \\
\text { - Falta de interactividad de los } \\
\text { contenidos formativos. } \\
\text { - Utilización de páginas } \\
\text { demasiado extensas, } \\
\text { que requieren del uso del } \\
\text { "ascensor", y dificultan su } \\
\text { consulta y navegación. }\end{array}$ & \begin{tabular}{|l} 
- No se establecen \\
claramente los criterios \\
de evaluación. \\
- No se explicita \\
nítidamente en qué \\
consisten las actividades \\
a desarrollar por los \\
discentes. \\
- Falta de planificación de \\
actividades de carácter \\
grupal en determinadas \\
asignaturas.
\end{tabular} & $\begin{array}{l}\text { - No se contempla el desarrollo } \\
\text { de sesiones de Chat en } \\
\text { la programación de las } \\
\text { asignaturas. } \\
\text { - Falta de comunicación, } \\
\text { interacción y participación de } \\
\text { los discentes. } \\
\text { - No se potencia la tutoría } \\
\text { individualizada. } \\
\text { - No se han generado } \\
\text { comunidades virtuales de } \\
\text { aprendizaje. }\end{array}$ \\
\hline
\end{tabular}




\begin{tabular}{|c|c|c|c|}
\hline \multicolumn{4}{|c|}{ DEBILIDADES DETECTADAS EN LAS ASIGNATURAS MATRIX POR ÁREAS DE CONOCIMIENTO } \\
\hline \multirow{2}{*}{$\begin{array}{c}\text { Área de } \\
\text { Conocimiento }\end{array}$} & \multicolumn{3}{|c|}{ Aspectos objeto de análisis } \\
\hline & $\begin{array}{c}\text { Diseño Técnico de los } \\
\text { Materiales }\end{array}$ & Diseño Instructivo & Acción Tutorial \\
\hline $\begin{array}{c}\text { Ciencias } \\
\text { Biológicas y } \\
\text { de la Salud }\end{array}$ & $\begin{array}{l}\text { - Utilización de un diseño en } \\
\text { el que no existe contraste } \\
\text { fondo/texto. } \\
\text { - Presentación de los } \\
\text { contenidos únicamente } \\
\text { en formato pdf para su } \\
\text { impresión. }\end{array}$ & $\begin{array}{l}\text { - No se contempla } \\
\text { la realización de } \\
\text { actividades de carácter } \\
\text { grupal. }\end{array}$ & $\begin{array}{l}\text { - No se desarrolla la acción } \\
\text { tutorial a través de las } \\
\text { herramientas presentes en } \\
\text { la plataforma (sino de forma } \\
\text { presencial exclusivamente). }\end{array}$ \\
\hline
\end{tabular}

Tabla 2. Debilidades de las asignaturas MATRIX, detectadas a partir de los análisis DAFO, considerando los diferentes aspectos objeto de estudio

Entre las fortalezas -detectadas por los evaluadores externos- se destacan, en lo relativo al diseño técnico de los materiales, su claridad en la presentación, el uso adecuado de diferentes recursos multimedia, la interactividad de los contenidos, la incorporación de un sistema de navegación intuitivo, etc... En lo que respecta al diseño instructivo, se valora positivamente la presencia de una guía didáctica, la formulación de actividades que propicien la participación, el uso de herramientas que faciliten el estudio, la coherencia entre los objetivos, contenidos, actividades y evaluación, etc... Y sobre la acción tutorial, se subraya la existencia de prácticas tutoriales individuales y grupales orientadas a la creación de comunidades de aprendizaje activas a partir de herramientas como foros, chat, wikis, etc...

Por su parte, las debilidades detectadas en algunas de las asignaturas coinciden en evidenciar la ausencia de un diseño técnico de los materiales didácticos adecuado, accesible, usable y claro, sin elementos interactivos, y con un sistema de navegación limitado. Un diseño instructivo poco coherente entre los objetivos y la evaluación elegida, así como la propuesta de actividades poco motivadoras. Y una actuación tutorial pobre, que no contribuye a fomentar la participación y comunicación entre los discentes.

\section{Presentación de los resultados cuantitativos más destacados: las opiniones de los estudiantes del CVC G9 obtenidos a través de un cuestionario}

Por otro lado, dentro de los objetivos del proyecto MATRIX, se consideró oportuno valorar las opiniones que los estudiantes universitarios tenían en relación a los 
contextos en los que desarrollaban su aprendizaje y, en concreto, sobre determinados aspectos ligados a los recursos didácticos que pueden contribuir a facilitarles el mismo; la orientación tutorial dispensada por los docentes en ellos; las habilidades que perciben poseer éstos para la gestión de la comunicación y el fomento de la participación en dichos contextos. Para ello, durante el segundo cuatrimestre del curso académico 2006/07 se aplicó un cuestionario a los estudiantes que cursaban alguna asignatura en el CVC del G9, para constatar sus valoraciones a este respecto.

Algunos de los datos obtenidos más representativos revelan que el 70\% de los discentes declaran que su nivel de satisfacción general en relación a la presentación y tutorización de las asignaturas que han cursado en su modalidad virtual ha sido "alto" o "muy alto". La mayoría de los estudiantes manifiestan que entre los factores que han condicionado su valoración positiva se halla el hecho de que los docentes responsables de cada asignatura mayoritariamente han facilitado el acceso a los materiales y las actividades didácticas mediante una organización y estructuración adecuada de los mismos, tal y como señala el $67 \%$ de los mismos. De igual manera, el $58 \%$ entienden que los docentes "siempre" o "casi siempre" han propuesto actividades que posibilitaron la asimilación de los contenidos, facilitando con ello su proceso de aprendizaje.

Por otro lado, algo más del $65 \%$ de los estudiantes ponen de relieve que los docentes de las asignaturas cursadas, mayoritariamente, han realizado pruebas o ejercicios de evaluación continua, valorándolo muy positivamente, puesto que ello tiene una repercusión directa en la permanencia del estudiante en el entorno virtual de aprendizaje al invitarle a seguir la asignatura con regularidad y a alcanzar con éxito los objetivos formativos; además de influir en su nivel de motivación intrínseca, proporcionándole un feed-back permanente sobre sus progresos en la asignatura en la que se encontraba matriculado.

También es preciso subrayar que aproximadamente el 40\% de los discentes declaran que gran parte de los docentes de las materias que cursaron fomentan el trabajo colaborativo mediante el planteamiento de diferentes actividades formativas. En este sentido, algo más del $43 \%$ de ellos consideran que "casi siempre" el profesor promueve la participación y comunicación entre los estudiantes, condición que consideramos imprescindible para garantizar el buen desarrollo de las actividades colaborativas planteadas.

A pesar del alto nivel de satisfacción y de los buenos resultados obtenidos en las valoraciones efectuadas por la mayoría de los discentes en relación tanto a la 
presentación de los contenidos y actividades didácticas propias de las asignaturas virtuales que han cursado, como a la acción tutorial dispensada por los docentes, no podemos dejar de señalar que el $11 \%$ de ellos manifiesta tener un nivel de satisfacción "bajo" o "muy bajo", dato que se encuentra directamente relacionado,-y en porcentajes similares-, con la consideración de los mismos sobre algunos aspectos claves e intrínsecos a la metodología didáctica adoptada por los docentes, ya que algo más del $17 \%$ de los discentes perciben que los docentes responsables de las asignaturas virtuales cursadas no aplican fórmulas de evaluación adecuadas y efectivas para constatar el nivel de asimilación de los contenidos y el logro de los objetivos. Igualmente, un $14 \%$ consideran que los contenidos no son lo suficientemente apropiados para la consecución de los objetivos formativos, dado que su estructura no les ha facilitado el estudio de los materiales incorporados en la materia. Los mismos manifiestan que los docentes de la asignatura que cursaron no presentan los objetivos formativos de forma clara y explícita, elementos indispensables y básicos que deberían tenerse en cuenta en toda acción formativa para guiar el proceso de enseñanza-aprendizaje.

Por otra parte, al preguntarles a los estudiantes por aspectos, tales como los vinculados a la función tutorial llevada a cabo por los docentes, hay que resaltar que la cuarta parte de ellos echan en falta que éstos se intenten adaptar a los conocimientos de partida que poseen, así como a sus peculiaridades cognitivas o estilos de aprendizaje propios. Otro 35\%, nada desdeñable, reclama que los tutores les proporcionen comentarios individualizados de apoyo y ánimo en relación a sus progresos en el aprendizaje. Ambas demandas pueden considerarse estrechamente relacionadas con su nivel de motivación para participar en las dinámicas generadas en las asignaturas.

Al solicitar a los estudiantes que valorasen las habilidades de los docentes para la gestión de la comunicación y la participación en esos entornos virtuales, se constata como más del $34 \%$ declara que al inicio de la materia no se les han propuesto actividades (foros de presentación...) destinadas a fomentar un primer contacto entre todos los integrantes de la misma con el objetivo de establecer un vínculo que propicie la interacción y favorezca la realización posterior de actividades formativas conjuntas, ayudando a consolidar la comunidad de aprendizaje de la propia asignatura. Cabe destacar, así mismo, que el 20\% de los discentes perciben que el feed-back llevado a cabo por el docente no es lo suficientemente ágil como les hubiera gustado, factores que consideramos de vital importancia, pues de ellos, a menudo, va a depender en gran medida la permanencia del estudiante en el entorno de la asignatura. 


\section{FORMULACIÓN DE INDICADORES DE CALIDAD EXTRAPOLABLES AL “BLENDED LEARNING" DERIVADOS DE LOS RESULTADOS}

Tomando como marco de referencia tanto los factores e indicadores de calidad para la educación en general, enunciados por diversos autores Sarramona (2004), Villar Angulo y Alegre de la Rosa (2004), etc..., como para los procesos formativos virtuales, en particular, Del Moral y Villalustre (2005), Marcelo (2008); y tras la exhaustiva evaluación de las asignaturas que han formado parte del proyecto MATRIX a partir de los análisis DAFO, realizados por los expertos; y las valoraciones vertidas por los estudiantes a través del cuestionario de opinión, se infieren las propuestas de mejora para cada una de ellas, las cuales pasan por la enumeración, de modo más preciso, de los indicadores de calidad que contribuyen a definir las "buenas prácticas" docentes apoyadas en entornos virtuales de forma total o parcialmente.

Como resultado de los procesos de análisis llevados a cabo a lo largo del mencionado proyecto, se han delimitado un conjunto de indicadores de calidad orientados a diseñar una propuesta de mejora para el éxito de las prácticas docentes que apuesten por una modalidad virtual y/o semipresencial, la cual contempla tres ejes fundamentales:

- Diseño técnico de materiales didácticos

- Diseño instructivo

- Acción tutorial

Indicadores de calidad relativos al diseño técnico de materiales didácticos

En el diseño de materiales didácticos para la formación virtual y semipresencial se considera necesario atender a un conjunto de factores que garanticen la calidad del escenario instructivo y, más específicamente, de su interfaz gráfica. Éstos deben contemplar los siguientes elementos:

\begin{tabular}{|c|c|}
\hline Diseño del interfaz & Iconos y mapas de navegación \\
\hline Metáforas del entorno & Presentación de la información \\
\hline
\end{tabular}

Gráfico 4. Elementos a tener presente a la hora de valorar el diseño técnico de los materiales didácticos 
Al diseñar un interfaz gráfico se deben tener presente diferentes criterios de usabilidad, accesibilidad, interactividad y adaptabilidad, tal y como apuntan Del Moral (1999, 2004), Krug (2001), Nielsen (2002), Cabero (2002) y Del Moral y Villalustre (2005), los cuales contemplen aspectos tan diversos como la organización de los contenidos, el diseño de elementos interactivos hipermedia, la concreción de diferentes niveles de interactividad y de accesibilidad, ..., etc. Puesto que tan importante es la calidad de la información que se presenta como la forma de presentarla y estructurarla. El propio formato visual de presentación y organización de la misma será el que propicie una mayor o menor interacción del estudiante con los materiales incorporados en el entorno, el cual condicionará en gran medida el que éste capte y, posteriormente, asimile aquella información que le resulte más atractiva visualmente.

Al igual que en la enseñanza presencial muchos discentes "on-line" ven su proceso formativo condicionado por el entorno virtual en el que se encuentran inmersos, provocando, en algunas ocasiones, el abandono de la acción instructiva al no sentirse cómodos con el formato visual, por este motivo, se considera prioritario ser rigurosos desde el punto de vista estético y técnico, y cumplir con las normas básicas de usabilidad, legibilidad, accesibilidad, etc... (Krug, 2001).

Igualmente, la estructuración de los contenidos didácticos facilita, sin duda, la exploración de la información, sin embargo, es necesario que ésta se encuentre organizada y se pueda llegar a ella a través de mecanismos de navegación eficaces y accesibles, los cuales faciliten el periplo del estudiante por el entorno virtual.

Por otro lado, la utilización de metáforas de fácil identificación por parte del estudiante en el entorno virtual de aprendizaje, facilitará la comprensión de la estructura y la asimilación de los diferentes mecanismos de interacción presentes en el mismo (Barker y Manji, 1991). Así, cuando la metáfora seleccionada es conocida por el aprendiz la comprensión del entorno queda garantizada, y simplifica la actividad cognitiva del discente, propiciando que el ambiente de aprendizaje no sea un obstáculo.

\section{Indicadores de calidad relativos al diseño instructivo}

Desde una perspectiva pedagógica se deben valorar aquellos elementos que se hallan directamente relacionados con el adecuado desarrollo cognitivo de los estudiantes, y que contribuyan a la optimización del proceso de enseñanzaaprendizaje que tiene lugar en contextos virtuales o semipresenciales. Entre los 
que cabe mencionarse: la delimitación a priori los objetivos de aprendizaje que van a constituir las metas formativas a alcanzar por los estudiantes; la adecuada formulación de actividades que permitan valorar el progreso de los estudiantes; la clarificación del sistema de evaluación para que éstos conozcan las criterios que se van a emplear, etc.

Es preciso partir de la base de que el diseño instruccional debe proporcionar a los estudiantes experiencias y ambientes de aprendizaje contextualizados a través de los cuales se favorezca la adquisición de conocimientos y el desarrollo de competencias de diversa índole. De ahí que, tanto con las actividades propuestas, como con los objetivos delimitados, los contenidos expuestos, etc..., se intente generar un espacio para potenciar los aprendizajes significativos, de modo que se propicie la extrapolación de dichos aprendizajes a experiencias similares.

\section{Indicadores de calidad relativos a la acción tutorial}

El papel que el tutor desempeña en los nuevos escenarios para formación virtual y/o semipresencial, ha de ser sustancialmente diferente al que venía ejerciendo en la enseñanza convencional, puesto que ahora debe asumir nuevas competencias que van a determinar el éxito del proyecto formativo. El apoyo y asesoramiento constante condicionan en gran medida la permanencia del estudiante en el entorno virtual de aprendizaje y su participación en las diferentes tareas propuestas.

La acción tutorial, en cualquier caso, debe tender a desarrollar al máximo las potencialidades de los estudiantes, facilitándoles los recursos necesarios para ello, orientándoles para subsanar de forma autónoma las dificultades que les surjan a lo largo de su proceso formativo, sin olvidar el marco contextual en el que se encuentran y atendiendo a las necesidades, motivaciones e intereses de cada uno de ellos. Una tarea que debe responder a una planificación cuidada y sistemática, capaz de propiciar la participación activa y efectiva de todos ellos, conjugando la confianza con la exigencia para optimizar las actividades formativas y el autoaprendizaje. Así, al docente que desempeña su actividad apoyado en el uso de entornos virtuales, no sólo se le pide que sepa transmitir los contenidos de su matera, sino también que posea habilidades en tanto orientador y facilitador del aprendizaje (Del Moral, 2004). 


\section{CONCLUSIONES}

La reformulación del modelo de enseñanza universitaria derivada de los cambios suscitados a partir del proceso de Convergencia Europa, supone un nuevo reto para la práctica pedagógica del docente al tener que planificar una enseñanza que garantice tanto el desarrollo integral del estudiante mediante la potenciación y consolidación de diferentes competencias, como la adquisición de habilidades relativas al uso eficiente de las herramientas informáticas, muy demandadas en la sociedad actual (González y Wagenaar, 2003).

Con el proyecto MATRIX se pretendía promover la reflexión sobre los criterios metodológicos, estrategias y actividades más adecuadas que deben adoptarse en las modalidades formativas mixtas (semipresenciales), y así contribuir a determinar las claves que garantizan la eficacia del quehacer educativo. En este sentido nos encontramos con que hoy, el reto de las enseñanzas universitarias radica en ofrecer escenarios formativos, tanto presenciales como virtuales, compatibles y complementarios que atiendan a las necesidades de los estudiantes al tiempo que se incorporan innovaciones pedagógicas que faciliten su desarrollo, según explica De Pablos (2008).

Las experiencias formativas apoyadas en entornos virtuales analizadas representan unas prácticas docentes innovadoras que, a juicio de los evaluadores externos, poseen numerosas fortalezas, entre las que cabe mencionarse las relativas al diseño técnico de los materiales didácticos que incorporan; la pluralidad de actividades tanto individuales como grupales que en ellas se incorporan para el desarrollo de competencias de diverso tipo; y las prácticas tutoriales, que se realizan para garantizar la orientación y la participación de los estudiantes.

Las valoraciones de una muestra de 290 estudiantes del CVC del G9, recabadas a través de un cuestionario en relación a su nivel de satisfacción general con las asignaturas cursadas, así como sobre determinados aspectos íntimamente relacionados con las competencias didácticas y tutoriales de los docentes responsables de las mismas, ponen de manifiesto que, si bien el 70\% de los discentes manifestaron que su nivel de satisfacción general era "alto o muy alto", y para un 19\% era "medio"; sin embargo, un nada desdeñable 11\% afirmaba no estar satisfecho (nivel bajo) con la metodología y formación recibida en las asignaturas virtuales que cursaron, dato que se encuentra directamente relacionado con su consideración sobre aspectos claves e intrínsicos a la metodología didáctica y tutorial adoptada por los docentes, tales como: 
- La presentación de unos objetivos formativos poco claros y explícitos.

- El diseño de unos contenidos poco apropiados para la consecución de los objetivos formativos.

- La utilización de fórmulas de evaluación poco adecuadas y efectivas para constatar el nivel de asimilación de los contenidos y el logro de los objetivos.

- La escasez de comentarios individualizados de apoyo y ánimo en relación a sus progresos en el aprendizaje (...), etc.

Así, tanto las mejoras formuladas por los expertos a partir de los análisis DAFO realizados, como las valoraciones de los estudiantes sobre los entornos virtuales en los que han desarrollado su aprendizaje, inciden en la necesidad de:

- Explicitar a priori los objetivos de aprendizaje que van a constituir las metas formativas a alcanzar por los estudiantes.

- Desarrollar y presentar los contenidos de forma jerárquica, contextualizada y coherentemente estructurada.

- Presentar diversidad de actividades tanto individuales como grupales a través de las cuales se pueda valorar el progreso de los estudiantes.

- Clarificar el sistema de evaluación, haciendo visibles los criterios de valoración acordes con los objetivos a lograr, que se van a emplear.

- Favorecer espacios para la tutoría individualizada que contribuyan a la orientación, el apoyo y la motivación de los estudiantes.

Todo ello puede servir de referente vicario para que los docentes universitarios puedan crear y diseñar sus asignaturas virtuales y/o semipresenciales con la garantía de saber que redundan en la eficacia del quehacer educativo.

\section{NOTAS}

1 Del Moral Pérez, M.E. (dir.) (2007): Proyecto MATRIX (Modalidades de Aprendizaje Telemático y Resultados Interuniversitarios eXtrapolables al blended learning. Financiado por el MEC EA2007, en el cual han participado 20 docentes procedentes de la Universidad de Oviedo, U. Pública de Navarra, U. de País Vasco, U. de Zaragoza y de la U. de Extremadura. 


\section{REFERENCIAS BIBLIOGRAFICAS}

Barker, P.; Manji, K. (1991). Designing Electronic Books. Educational and Learning Technology International (ETTI). 28 (4).

Bogdan, R.; Biklen, S. (1982). Qualitative Research for Education: An Introduction to Theory and Methods. Boston: Allyn and Bacon.

Cabero, J. (Dir.) (2002). Diseño y Evaluación de un Material Multimedia y Telemático Para La Formación y Perfeccionamiento del Profesorado Universitario para la utilización de las Nuevas Tecnologías Aplicadas a la Docencia. Sevilla: Universidad de Sevilla.

De Pablos Pons, J. (2008). Nuevas formas de trabajo en las aulas universitarias con el soporte de las Tecnologías de la Información y Comunicación. En: Del Moral, M. E.; Rodríguez, R. Experiencias docentes y TIC. Barcelona: Editorial Octaedro e ICE Universidad de Oviedo. (43-58).

Del Moral, M. E. (1999). Metáforas, recursos interactivos y ambientes hipermedia para el aprendizaje. I Jornadas de Multimedia Educativo. Barcelona. 5-7 julio.

Del Moral, M. E. (2004). Redes como soporte a la docencia. Tutoría online y aplicaciones telemáticas. En: Rodríguez, R. y otros. Docencia Universitaria. Orientaciones para la formación del profesorado. Oviedo: Universidad de Oviedo. (193-212).

Del Moral, M. E.; Villalustre, L. (2005). Indicadores de calidad para un interfaz gráfico centrado en el aprendiz. $V$ Congreso Internacional Virtual de Educación (CIVE). Unisersitat de les Illes Baleares. 7 al 27 de febrero.

Del Moral, M. E.; Villalustre, L. (2008). Desarrollo de competencias y estilos de aprendizaje en contextos virtuales: prácticas colaborativas y trabajo autónomo en Ruralnet. En: Del Moral, M.E. y Rodríguez, R. (coords). Docencia Universitaria. Experiencias docentes y TIC. ICE de la Universidad de Oviedo: Editorial Octaedro. (97-129).

Del Moral, M. E.; Cernea, D. A.; Villalustre, L. (2007). Contributions of the Web 2.0 to collaborative work around Learning Objets. In Proceeding of the International Conference on Technology, Training and Communication. Salamanca, Septiembre 12-14. [en línea] Disponible en: http://ftp.informatik.rwth-aachen. de/Publications/CEUR-WS/Vol-361/ paper13.pdf [consulta 2009, 10 de Febrero]

Del Moral, M.E. (2004). Sistemas Interactivos Hipermedia Educativos. En: Del Moral, M. E. (coord.). Sociedad del conocimiento, ocio y cultura: un enfoque interdisciplinar. Oviedo: Ediciones KRK.

Dodge, B. (1995). Some Thoughts About WebQuest. [en línea] Disponible en: http://webquest.sdsu.edu/about webquests.html. [consulta 2008, 20 de Junio]

Epper, R.; Bates, A. W. (2004). Enseñar al profesorado como utilizar la tecnología. Buenas prácticas de instituciones líderes. Barcelona: Editorial UOC.

Goetz, J. P.; Lecompte, M. D. (1988). Etnografía y diseño cualitativo en investigación educativa. Madrid: Morata.

González, J.; Wagenaar, R. (Coor.) (2003). TUNING. Educational Strutures in Europa. Informe Final del Proyecto Piloto - Fase 1. Bilbao: Universidad de Deusto.

Krug, S. (2001). No me hagas pensar. Una aproximación a la usabilidad en web. Madrid: Editorial Person Educación. 
Marcelo, C. (2008). Evaluación de la calidad para programas completos de formación docente a través de estrategias de aprendizaje abierto y a distancia. Revista de Educación a Distancia, año VIII, $\mathrm{n}^{\mathrm{o}}$ monográfico VII, diciembre.

Nilsen, J. (2002). Usabilidad. Diseño de sitios web. Madrid: Editorial Person Educación.
Sarramona, J. (2004). Factores e indicadores de calidad en la educación. Barcelona: Octaedro.

Villar Angulo, L. M.; Alegre De la Rosa, O. M. (2004). Manual para la excelencia en la Enseñanza Superior. Madrid: McGrawHill.

\section{PERFIL ACADÉMICO Y PROFESIONAL DE LAS AUTORAS}

Ma Esther del Moral Pérez. Catedrática de E.U. en NNTT aplicadas a la Educación en la Universidad de Oviedo. Coordinadora del Grupo de investigación Tecn@: Tecnología y aprendizaje. Docente de la asignatura "Educación en el ámbito rural" impartida "on line" dentro del Campus Virtual Compartido del G9. Entre sus publicaciones destacan: Experiencias Docentes y TIC (2008), Sociedad del Conocimiento: ocio y cultura (2004), Reflexiones sobre NNTT y Educación (1998). Ha escrito varios capítulos de libros en colaboración con otros autores y numerosos artículos en revistas. Así mismo, posee numerosas comunicaciones en Congresos Nacionales e Internacionales.

E-mail: emoral@uniovi.es

Lourdes Villalustre Martínez. Maestra y Licenciada en Pedagogía por la Universidad de Oviedo. Su actividad investigadora se centra en el estudio de entornos virtuales de aprendizaje y estilos cognitivos, así como en el diseño de aplicaciones formativas hipermedia, como miembro del grupo de investigación “Tecn@”. Ha publicado artículos en varias revistas y cuenta con numerosas aportaciones en Congresos Nacionales e Internacionales. Ha participado en diversos proyectos de investigación financiados por el MEC, la Universidad de Oviedo, etc.

E-mail: villalustrelourdes@uniovi.es

DIRECCIÓN DE LAS AUTORAS

Facultad de CC de la Educación

Universidad de Oviedo

$\mathrm{C} /$ Aniceto Sela, s/n

33005 Oviedo (Asturias)- España

Fecha de recepción del artículo: 18/06/08

Fecha de aceptación del artículo: 10/02/09 